\title{
Contribution of birth weight to mental health, cognitive and socioeconomic outcomes: two-sample Mendelian randomisation
}

Massimiliano Orri, Jean-Baptiste Pingault, Gustavo Turecki, Anne-Monique Nuyt, Richard E. Tremblay, Sylvana M. Côté and Marie-Claude Geoffroy

\section{Background}

Low birth weight is associated with adult mental health, cognitive and socioeconomic problems. However, the causal nature of these associations remains difficult to establish owing to confounding

\begin{abstract}
Aims
To estimate the contribution of birth weight to adult mental health, cognitive and socioeconomic outcomes using two-sample Mendelian randomisation, an instrumental variable approach strengthening causal inference.
\end{abstract}

\section{Method}

We used 48 independent single-nucleotide polymorphisms as genetic instruments for birth weight (genome-wide association studies' total sample: $n=264498$ ) and considered mental health (attention-deficit hyperactivity disorder (ADHD), autism spectrum disorder, bipolar disorder, major depressive disorder, obsessivecompulsive disorder, post-traumatic stress disorder (PTSD), schizophrenia, suicide attempt), cognitive (intelligence) and socioeconomic (educational attainment, income, social deprivation) outcomes.

\section{Results}

We found evidence for a contribution of birth weight to ADHD (OR for 1 s.d. unit decrease ( 464 g) in birth weight, 1.29; 95\% $\mathrm{Cl} 1.03-1.62)$, PTSD (OR $=1.69 ; 95 \% \mathrm{Cl} 1.06-2.71)$ and suicide attempt (OR $=1.39 ; 95 \% \mathrm{Cl} 1.05-1.84)$, as well as for intelligence $(\beta=-0.07 ; 95 \% \mathrm{Cl}-0.13$ to -0.02$)$ and socioeconomic outcomes, i.e. educational attainment $(\beta=-0.05 ; 95 \% \mathrm{Cl}-0.09$ to -0.01 ), income ( $\beta=-0.08 ; 95 \% \mathrm{Cl}-0.15$ to -0.02 ) and social deprivation ( $\beta=0.08 ; 95 \% \mathrm{Cl} 0.03-0.13$ ). However, no evidence was found for a contribution of birth weight to the other examined mental health outcomes. Results were consistent across a wide range of sensitivity analyses.

\section{Conclusions}

These findings support the hypothesis that birth weight could be an important element on the causal pathway to mental health, cognitive and socioeconomic outcomes.

\section{Keywords}

Psychiatric disorders; birth weight; Mendelian randomisation; socioeconomic outcomes; intelligence.

\section{Copyright and usage}

(c) The Author(s), 2021. Published by Cambridge University Press on behalf of the Royal College of Psychiatrists.
Low birth weight (a global index of poor fetal development) has been associated with a range of mental health problems (including attention-deficit hyperactivity disorder (ADHD), autism, bipolar disorder, depression, schizophrenia and suicide), ${ }^{1-8}$ as well lower intelligence and socioeconomic status ${ }^{9-11}$ (see also the Introduction in the supplementary material available at https://doi.org/10.1192/bjp.2021.15). These findings are consistent with the developmental origins of health and disease (DOHaD) hypothesis, ${ }^{12,13}$ which states that adverse in utero and perinatal experiences may have long-lasting effects on adult health. Yet, the causal nature of these associations remains unclear. Birth weight is influenced by a range of intrauterine exposures and maternal conditions and behaviours, such as mental health and diet, exposure to tobacco and alcohol, toxins, pollution and socioeconomic adversity. ${ }^{14-20}$ Those factors are likely to confound the association between birth weight and mental health and socioeconomic outcomes, because such confounding factors may cause a change in both birth weight and outcomes. Clarifying whether birth weight is a causal risk factor for mental, cognitive and socioeconomic problems is important for understanding their aetiology. Given that it is not possible to directly randomise birth weight to probe its causal role on later outcomes, the most robust evidence would come from quasi-experimental designs. Mendelian randomisation is a methodology that strengthens causal inference on the association between an exposure and an outcome using genetic variants as instruments. ${ }^{21-23}$ Genetic variants are randomly allocated at conception and are relatively independent of environmental confounding factors; therefore this design mimics that of a randomised trial in which treatment is randomly allocated and confounding factors do not depend on treatment allocation (Fig. 1; see supplementary material Methods for details on Mendelian randomisation assumptions). ${ }^{21,22}$ A previous study that used Mendelian randomisation to investigate the role of birth weight in ADHD, major depressive disorder and schizophrenia found no evidence for a causal role of birth weight. ${ }^{24}$ However, a major limitation of that study was the inability to account for the confounding effect of maternal genotype, which can lead to incorrect Mendelian randomisation estimates. ${ }^{25,26}$ Maternal and individual (i.e. offspring) genotypes are correlated and any effect of intrauterine exposures or maternal behaviour influenced by the mother's genetic make up may also result in an association between the offspring's genotype and mental health outcomes (Fig. 1). However, new data from a recently published genome-wide association study (GWAS) of birth weight ${ }^{26}$ providing estimates of the association of singlenucleotide polymorphisms (SNPs) with birth weight after adjustment for the correlation between maternal and individual genotypes enable us, for the first time, to overcome this limitation. The present Mendelian randomisation study relies on summary statistics from the largest available GWASs to estimate the contribution of birth weight to mental health (including common psychiatric disorders and suicide attempt), cognitive (i.e. intelligence) and socioeconomic outcomes (including educational attainment, income and social deprivation). 


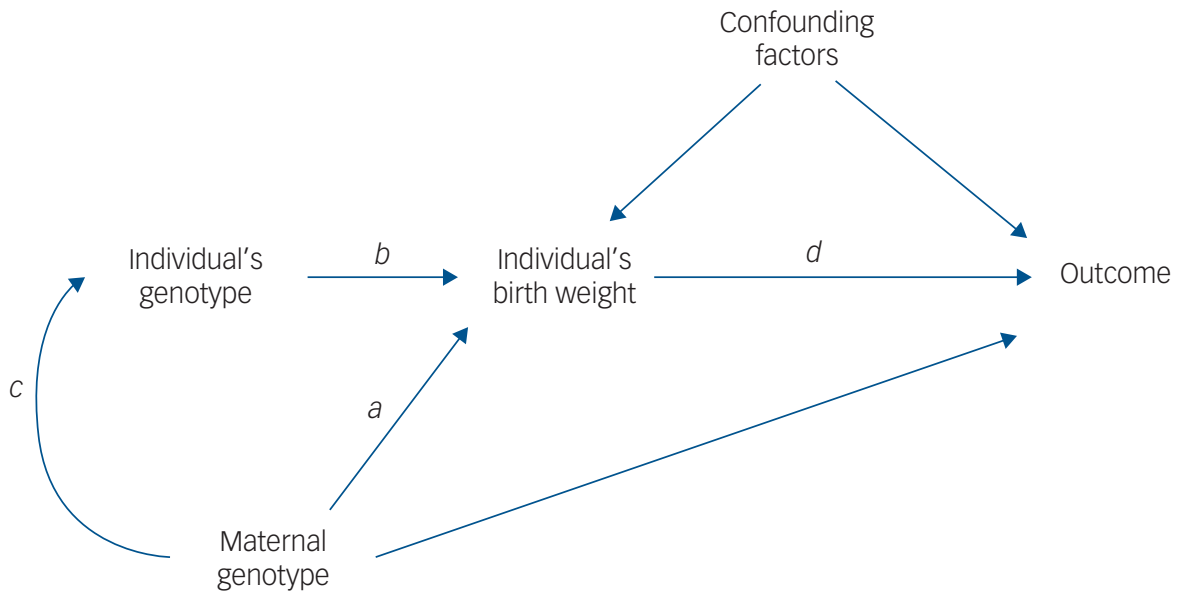

\section{Fig. 1 Confounding effect of maternal genotype on the association between an individual's genotype and birth weight.}

Using the Mendelian randomisation design, it is possible to estimate the association between an individual's birth weight and an outcome (path $d$ in the figure) using the individual's genotype associated with birth weight as the instrumental variable (path $b$ ), instead of the observational assessment of birth weight. The association estimated in this way is not confounded by factors (such as maternal substance use) that may confound the association between birth weight and outcome in observational studies. However, this design alone does not take into account the confounding effect of maternal genotype. Indeed, both the individual's genotype (path $a$ ) and maternal genotype (path $b$ ) have influences on birth weight, the former directly, the latter through the intrauterine environment. Because of the correlation between the individual's genotype and their mother's genotype $(r \sim 0.5$; path c), the effect of the individual's phenotype on their birth weight may be confounded. To avoid this bias, we used estimates of the association between individuals' genetic variants adjusted for the correlated maternal effect as instruments (published in the most recent birth-weight GWAS). ${ }^{26}$

\section{Method}

\section{Data sources}

This study relied on summary statistics from GWASs performed by international consortia (Table 1). Only GWASs of individuals of European ancestry were used, as genetic variants can be differently associated with a trait in different ancestry groups owing to specific linkage disequilibrium structures. ${ }^{27}$ All the GWASs had been adjusted for population stratification using ancestry-informed principal components, as well as for other main covariates (e.g. age and gender; see details in cited publications). All phenotypes were primarily measured among adult individuals and summary statistics were available only for both genders combined. We used the largest available non-overlapping exposure and outcome GWASs whenever possible, i.e. for all outcomes except for ADHD, intelligence and socioeconomic outcomes. However, this overlap is unlikely to bias the results (supplementary Methods). Power analysis is presented in the online material (supplementary Methods).

\section{Birth weight}

In total, $n=209$ independent genome-wide significant SNPs associated with birth weight were identified by the largest GWAS meta-analysis conducted by the Early Growth Genetics (EGG) consortium and including the UK Biobank sample $(n=264498){ }^{26}$

Table 1 Summary of genome-wide association studies used in the analyses

\begin{tabular}{|c|c|c|c|c|c|c|}
\hline \multirow[b]{2}{*}{ Phenotype } & \multirow{2}{*}{$\begin{array}{l}\text { Source GWAS or } \\
\text { consortium }\end{array}$} & \multicolumn{3}{|c|}{ Sample size, $n$} & \multirow[b]{2}{*}{ SNPS, $n$} & \multirow[b]{2}{*}{ Phenotype assessment } \\
\hline & & Total & Cases & Controls & & \\
\hline Birth weight & EGG, UKB & 264498 & - & - & 48 & Medical records, self-reports, midwife reports \\
\hline ADHD & PGC, IPSYCH, EAGLE & 53293 & 19099 & 34194 & 42 & $\begin{array}{l}\text { Registry-based diagnoses, self-reports, diagnostic } \\
\text { interviews }\end{array}$ \\
\hline Autism spectrum disorder & PGC, iPSYCH & 46350 & 18381 & 27969 & 44 & Registry-based diagnoses, clinical assessment \\
\hline Bipolar disorder & PGC & 46582 & 20352 & 31358 & 46 & $\begin{array}{l}\text { Diagnostic interviews, clinician-administered } \\
\text { checklists, medical records }\end{array}$ \\
\hline Major depressive disorder & PGC & 173005 & 59851 & 113154 & 46 & $\begin{array}{l}\text { Register-based diagnoses, diagnostic interviews, } \\
\text { questionnaires }\end{array}$ \\
\hline $\begin{array}{l}\text { Obsessive-compulsive } \\
\text { disorder }\end{array}$ & IOCDF-GC, OCG-AS & 9725 & 2688 & 7037 & 42 & DSM-IV diagnosis \\
\hline $\begin{array}{l}\text { Post-traumatic stress } \\
\text { disorder }\end{array}$ & PGC & 9537 & 2424 & 7113 & 46 & Diagnostic interviews, questionnaires \\
\hline Schizophrenia & CLOZUK, PGC & 105318 & 40675 & 64643 & 44 & Clinical assessment, diagnostic interviews \\
\hline Suicide attempt & iPSYCH & 50264 & 6024 & 44240 & 35 & Register-based ascertainment \\
\hline Intelligence & SSGAC & 269867 & - & - & 46 & Neurocognitive tests \\
\hline Educational attainment & SSGAC & 1131881 & - & - & 46 & Self-report \\
\hline Income & UKB & 96900 & - & - & 47 & Self-report \\
\hline Social deprivation & UKB & 112005 & - & - & 47 & Townsend deprivation index ${ }^{a}$ \\
\hline
\end{tabular}


Among these GWAS significant variants, we selected 48 SNPs identified as having an effect on birth weight after adjusting for the correlated maternal effect on birth weight, ${ }^{25}$ and maintaining statistical significance at $P<1 \times 10^{-5}$. The mean $F$-statistic for these SNPs was 36 (median, 28; range, 19-182; supplementary Methods), suggesting that all SNPs were strong instruments according to the suggested threshold of $F>10 .^{28}$ Birth weight (which had a mean of $\sim 3407 \mathrm{~g}$ and standard deviation of $\sim 464 \mathrm{~g}$ ) was $z$-score transformed separately for males and females in the studies participating in the GWAS meta-analysis and adjusted for study-specific covariates, including gestational duration (where available).

\section{Outcomes}

We obtained the estimates of associations between the birth weight instrument SNPs and our outcomes from the GWAS summary statistics. Whenever possible, instrument SNPs that were unavailable in the GWAS summary statistics of the outcome phenotype were replaced with overlapping proxy SNPs in linkage disequilibrium $\left(r^{2}>0.80\right)$ identified using the LDproxy online tool (https://ldlink. nci.nih.gov/). The following outcomes were considered: (a) mental health outcomes (all binary variables): ADHD, ${ }^{29}$ autism spectrum disorder, ${ }^{30}$ bipolar disorder, ${ }^{31}$ major depressive disorder, ${ }^{32}$ obsessive-compulsive disorder, ${ }^{33}$ post-traumatic stress disorder (PTSD), ${ }^{34}$ schizophrenia ${ }^{35}$ and suicide attempt (i.e. hospital admission for a suicide attempt); ${ }^{36}$ (b) cognitive outcome: intelligence (measured as the general factor of intelligence $(g)$ and primarily evaluating fluid domains of cognitive functioning); ${ }^{37}$ (c) socioeconomic outcomes: educational attainment (measured as years of education) ${ }^{38}$ household income (measured as total income before taxes using five income categories) ${ }^{39}$ and social deprivation (measured using the Townsend Social Deprivation Index). ${ }^{39}$ Details on phenotype assessment can be found in the individual publications.

\section{Data analysis}

We conducted a two-sample Mendelian randomisation analysis in $\mathrm{R}$ version 3.6 for $\mathrm{Mac}^{40}$ using the TwoSampleMR, ${ }^{41}$ MendelianRandomization ${ }^{42}$ and MRPRESSO packages. In twosample Mendelian randomisation, causal estimates can be obtained using summary statistics from different samples (i.e. GWASs), one for the instrument/SNP-exposure association, another for the instrument/SNP-outcome association. The two data-sets were harmonised and the positive strand alleles were inferred using allele frequencies for palindromes (minor allele frequency up to 0.4 ) whenever possible. Analyses including and excluding the remaining palindromic SNPs were conducted, yielding consistent results. Therefore, we reported results using the full set of SNPs. For each SNP, the ratio between the SNP-exposure and the SNP-outcome association (Wald test) was calculated. Then, Wald estimates for single SNPs were combined using random-effect inverse-variance weighting (IVW) meta-analysis as the primary analysis. This method corresponds to a weighted regression of SNP-outcome effects on SNP-exposure effects, in which weights were based on a multiplicative random-effects model. Heterogeneity across the meta-analysed estimates, which may be indicative of horizontal pleiotropy (i.e. the fact that the same SNPs influence multiple traits, so the association between instrument SNPs and outcome could not be entirely explained by the exposure, but act through alternative pathways, violating instrumental variable assumptions) ${ }^{22}$ was quantified using the $Q$-statistic (a significant test suggests pleiotropy).

A range of analyses were used to test the sensitivity of the IVW estimation. First, Mendelian randomisation-Egger (MR-Egger) regression, ${ }^{43}$ which relaxes the assumptions of Mendelian randomisation allowing for unbalanced pleiotropic effects. A major drawback of MR-Egger is the low power of this test; however, consistency in the direction and the size of the effect between the MREgger estimate and the IVW estimate can support the validity of the IVW analysis. We also used the intercept of the MR-Egger regression to test for the presence of unbalanced pleiotropy (a significant test suggests unbalanced pleiotropy). Second, we used the weighted median, which assumes that at least $50 \%$ of the total weight of the instrument comes from valid variants. It is more likely to give a valid causal estimate than the MR-Egger or the IVW method because it is more consistent with the true causal effect in the presence of up to $50 \%$ invalid variants. Third, we used the robust adjusted profile score (RAPS), ${ }^{44}$ which is an estimator that deals with weak instruments and is robust to pleiotropic effects.

We then performed four further analyses. First, MR-PRESSO (Mendelian Randomisation Pleiotropy Residual Sum and Outlier $)^{45}$ was used to detect and correct for outliers that may reflect bias due to pleiotropy. Second, leave-one-out analyses, in which the analyses were repeated by excluding one SNP instrument at a time, were performed to identify whether a single SNP was driving the association. Outlier SNPs were excluded from the analysis. Third, we searched the PhenoScanner database (a curated database of publicly available results from large-scale genetic association studies) for each SNP instrument (and those in linkage disequilibrium within $\left.r^{2} \geq 0.80\right)$ to see whether they have been associated $\left(P<1 \times 10^{-5}\right)$ with traits likely to bias our analysis because of horizontal pleiotropy or because of their association with confounders of the exposure-outcome association. In that case, these SNPs would be excluded in sensitivity analyses. Fourth, we conducted a Steiger filtering analysis to investigate whether the specified direction of the association (birth weight predicting mental health, cognitive and social outcomes) is further supported.

Associations were considered statistically significant at $P<0.05$. Additionally, to account for the possibility of false-positive findings, we used the false discovery rate with a $q$-value $<0.05$.

\section{Ethical approval}

This study is based on publicly available summary statistics from studies that had already obtained ethical approval; therefore, a separate ethical approval was not required.

\section{Results}

\section{Contribution of birth weight to mental health outcomes}

We found evidence for a contribution of birth weight to ADHD, with an OR of 1.29 (95\% CI 1.03-1.62; $P=0.027 ; q<0.05)$ per $1 \mathrm{~s}$. d. unit decrease in birth weight (Fig. 2). No evidence of horizontal pleiotropy was detected (MR-Egger intercept, $P=0.653$; supplementary Table 4$)$, but the $Q$-statistic indicated the presence of significant heterogeneity $(P=0.002)$. However, the association was consistent across the Mendelian randomisation methods used as sensitivity analyses (MR-RAPS OR $=1.27$; 95\% CI 1.01$1.61 ; P=0.045$; weighted median $\mathrm{OR}=1.34 ; 95 \%$ CI $1.00-1.81$; $P=0.054$; MR-Egger $\mathrm{OR}=2.11 ; 95 \%$ CI $1.31-3.34 ; P=0.001)$ and the MR-PRESSO and leave-one-out procedures did not detect any outlier. Similarly, we found evidence for a contribution of birth weight to PTSD (OR $=1.69 ; 95 \%$ CI $1.06-2.71 ; P=0.029$; $q<0.05$ ), with consistent estimates across sensitivity analysis methods (MR-RAPS OR $=1.71 ; 95 \%$ CI $1.02-2.88 ; P=0.044$; weighted median $\mathrm{OR}=2.09 ; 95 \%$ CI $0.98-4.44 ; \quad P=0.056$; MR-Egger OR $=3.00 ; 95 \%$ CI 0.96-9.38; $P=0.050)$ and no evidence for heterogeneity ( $Q$-statistic, $P=0.481$ ), unbalanced horizontal pleiotropy (MR-Egger intercept, $P=0.957$ ) and outliers influencing 


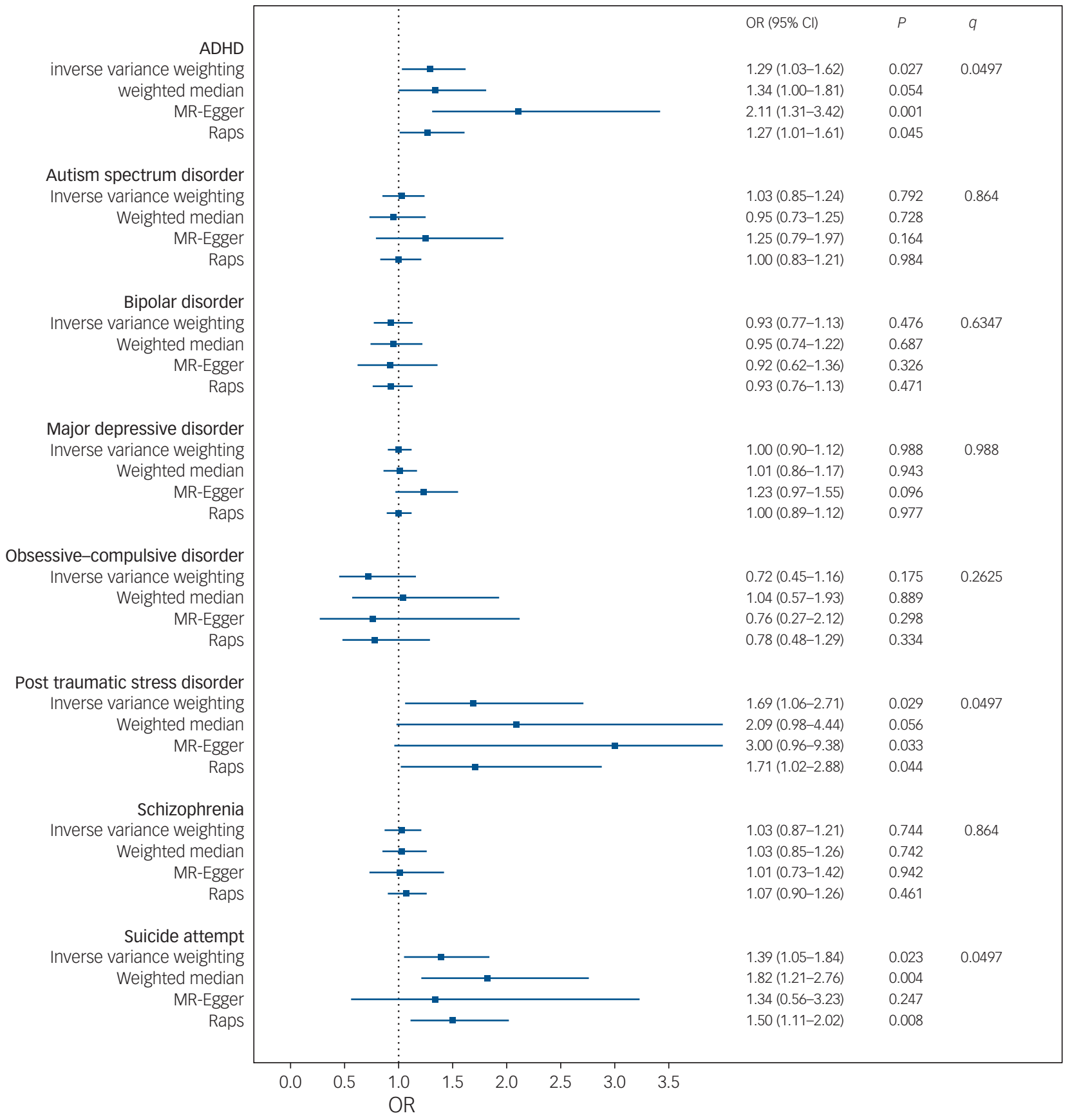

Fig. 2 Mendelian randomisation estimates for the association of birth weight with mental health. ADHD, attention-deficit hyperactivity disorder; MR-Egger, Mendelian randomisation-Egger regression; RAPS, robust adjusted profile score; $q, q$-value from the false discovery rate.

the results. We found no evidence supporting a contribution of birth weight to other psychiatric disorders, including autism spectrum disorder $(\mathrm{OR}=1.03 ; 95 \% \mathrm{CI} 0.85-1.24 ; P=0.792)$, bipolar disorder ( $\mathrm{OR}=0.93 ; 95 \% \mathrm{CI} 0.77-1.13 ; P=0.476)$, major depressive disorder (OR $=1.00 ; 95 \%$ CI $0.90-1.12 ; P=0.988)$, obsessive-compulsive disorder $(\mathrm{OR}=0.72 ; 95 \%$ CI $0.45-1.16 ; P=0.175)$ and schizophrenia $(\mathrm{OR}=1.08$; $95 \%$ CI $0.91-1.28 ; P=0.386)$. No unbalanced horizontal pleiotropy was detected for these outcomes; correcting for outlier SNPs detected for schizophrenia (rs1547669 and rs222857) did not alter the results. Furthermore, we found evidence supporting a contribution of birth weight to suicide attempt $(\mathrm{OR}=1.39$; $95 \%$ CI $1.05-1.84 ; P=0.023 ; q<0.05)$. Consistent results were found in sensitivity analyses (MR-RAPS OR $=1.50$; $95 \%$ CI 1.11 2.02; $P=0.008$; weighted median $\mathrm{OR}=1.82 ; 95 \%$ CI $1.21-2.76$;
$P=0.004$; MR-Egger $\mathrm{OR}=1.34 ; 95 \% \mathrm{CI} 0.56-3.23 ; P=0.247)$ and we did not find evidence for heterogeneity ( $Q$-statistic, $P=0.590$ ), unbalanced horizontal pleiotropy (MR-Egger intercept, $P=0.172$ ) and outliers.

\section{Contribution of birth weight to intelligence}

We found evidence for a contribution of birth weight to intelligence ( $\beta=-0.07 ; 95 \%$ CI -0.13 to $-0.02 ; P=0.010 ; q=0.001 ;$ Fig. 3 ) after exclusion of one outlier SNP (rs1482852; supplementary Results). This result remained after correction for an additional outlier SNP detected by the MR-PRESSO procedure (rs4144829; $\beta=-0.05 ; 95 \%$ CI -0.11 to $-0.01 ; P=0.036$ ). We did not find evidence for unbalanced horizontal pleiotropy (MR-Egger intercept, 


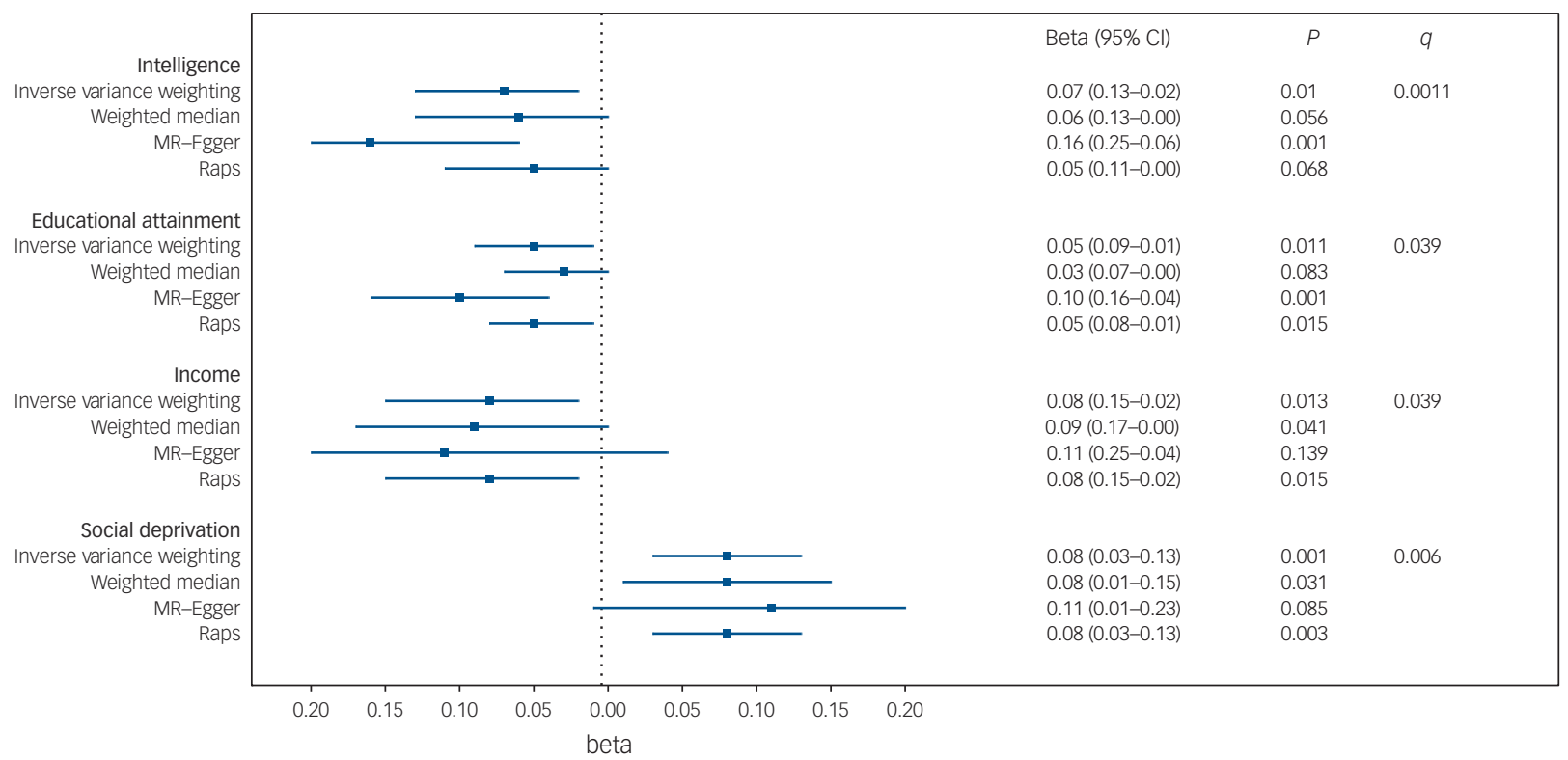

Fig. 3 Mendelian randomisation estimates for the association of birth weight with intelligence and socioeconomic outcomes. MR-Egger, Mendelian randomisation-Egger regression; RAPS, robust adjusted profile score; $q$, $q$-value from the false discovery rate.

$P=0.123)$, although there was significant heterogeneity according to the $Q$-statistic $(P<0.001)$.

\section{Contribution of birth weight to socioeconomic outcomes}

We found evidence for a contribution of birth weight to educational attainment ( $\beta=-0.05 ; 95 \% \mathrm{CI}-0.09$ to $-0.01 ; P=0.011 ; q=0.039)$, income $(\beta=-0.08 ; 95 \% \mathrm{CI}-0.15$ to $-0.02 ; P=0.013 ; q=0.039)$ and social deprivation ( $\beta=0.08 ; 95 \%$ CI $0.03-0.13 ; P=0.001 ; q=0.006$ ) (Fig. 3). MR-PRESSO detected outlier SNPs only for educational attainment (rs112139215, rs1129156, rs11698914, rs222857, rs4144829, rs7402983, rs7968682, rs8756), but outlier correction did not alter the results ( $\beta=-0.08 ; 95 \% \mathrm{CI}-0.08$ to $-0.02 ; P=0.005$ ). Educational attainment showed significant heterogeneity ( $Q$-statistic, $P<0.001)$. For income, we found evidence of both significant heterogeneity ( $Q$-statistic, $P=0.011$ ) and unbalanced pleiotropy (MR-Egger intercept, $P=0.024$ ), but all sensitivity analyses yielded consistent results (weighted median: $\beta=-0.09,95 \%$ CI -0.17 to $-0.00 ; P=0.041$; MR-Egger: $\beta=-0.11 ; 95 \% \mathrm{CI}-0.25$ to 0.04 ; $P=0.139$; MR-RAPS, $\beta=-0.08 ; 95 \%$ CI -0.15 to $-0.02 ; P=0.015$ ).

\section{Additional sensitivity analyses}

Searching the PhenoScanner database for each SNP instrument revealed associations between these SNPs and other anthropometric (e.g. height), metabolic (e.g. basal metabolism), hypertensive (e.g. blood pressure) and lipoprotein (e.g. high-density lipoproteins) traits. It is unlikely that those traits could generate bias by violating instrumental variable assumptions. Steiger filtering analyses suggested that the genetic variants used were indeed instruments for the exposure rather than for the outcomes (supplementary Results).

\section{Discussion}

Using a genetically informed instrumental variable approach to strengthen causal inference, this study investigated the contribution of birth weight to common psychiatric disorders, suicide attempt, and cognitive and socioeconomic outcomes. We found evidence supporting a role of birth weight in the pathway leading to ADHD, PTSD, suicide attempt, intelligence and socioeconomic outcomes (i.e. educational attainment, income and social deprivation), but not to the other examined mental health outcomes.

This study relied on a robust two-sample Mendelian randomisation design, the largest available GWAS summary statistics and multiple genetic instruments indexing birth weight. These features allowed our analyses to be well powered and to limit weak instrument bias. ${ }^{28}$ Furthermore, an innovative methodological feature is the use of genetic instruments adjusted for the correlated effect of maternal genotype. This approach has been previously applied to cardiometabolic outcomes ${ }^{26}$ but, to our knowledge, this is the first study relying on adjusted estimates to investigate the association of birth weight with mental health, cognitive and socioeconomic outcomes. As recently shown, ${ }^{25,26}$ failure to account for this confounding effect may create bias in the causal estimates.

Previous observational, ${ }^{46,47}$ within-sibling ${ }^{7}$ and twin $^{48}$ studies suggested an association between low birth weight and ADHD. Consistently, our results also suggest a potential causal role of birth weight in the aetiology of ADHD. ${ }^{7,48}$ Both ADHD and autism spectrum disorder are neurodevelopmental disorders with childhood onset and both had been associated with low birth weight. ${ }^{7}$ However, our study found evidence for potentially causal contribution of birth weight only to ADHD, suggesting that the contribution of birth weight might be specific to ADHD rather than common to neurodevelopmental disorders. This suggestion deserves further investigations, especially in light of a recent genetically informed (within-sibling) study showing associations with both ADHD and autism, as well as with a common neurodevelopmental latent factor. ${ }^{7}$ Future GWASs of autism, with larger sample size, will also provide the opportunity to re-test the association between birth weight and autism with a more powered analysis.

We found evidence supporting a potential causal role of birth weight on suicide attempt, consistent with a recent meta-analysis ${ }^{8}$ but not with a within-sibling Swedish study, ${ }^{49}$ which failed to find an association of birth weight with suicide attempt in early adulthood. Differences between the studies' populations (including age at suicide attempt assessment) and statistical power may explain these divergences. It is worth noting that we did not find evidence 
for a contribution of birth weight to depression, the psychiatric disorder that most strongly relates to suicide. ${ }^{50}$ As suicide risk is the result of both specific factors and factors shared with major psychiatric disorders comorbid with suicide, ${ }^{51}$ our finding points to birth weight as a factor causally contributing to suicide risk beyond factors also associated with depression. To further probe the role of birth weight in the aetiology of suicide, our finding needs to be replicated using suicide mortality, rather than suicide attempt, as an outcome. This will be possible when large-scale GWASs for suicide mortality become available.

Similarly, the documented association between birth weight and PTSD was in line with observational evidence on stress-related disorders, ${ }^{52}$ but not with a within-sibling study. ${ }^{7}$ However, the literature on this association is scarce and additional studies are needed.

Our study could not support the contribution of birth weight to other psychiatric disorders, including depression, bipolar disorder, obsessive-compulsive disorder and schizophrenia. These findings, in line with those of other quasi-experimental studies, ${ }^{7}$ are important, especially considering that available observational evidence was either contradictory (e.g. for depression $)^{5,53}$ or suggested associations (e.g. for schizophrenia). ${ }^{1}$

It is important to note that our study does not support a widespread contribution of birth weight to the general risk of psychopathology (i.e. the $P$-factor), but rather specific contributions to ADHD, PTSD and suicide attempt risk. However, future Mendelian randomisation investigations designed to specifically address this hypothesis may be informative to clarify the potential contribution of birth weight to common versus specific psychopathology factors. This effort may be facilitated by reliance on continuously measured outcomes (i.e. considering liability to psychopathology as a continuum) rather than on dichotomous outcomes as in the present study.

Inconsistent observational evidence was also available for the association of birth weight with socioeconomic outcomes, with some studies showing adult negative outcomes for low birthweight children compared with normal birth-weight children but others showing no differences. ${ }^{9,10}$ Our findings across various socioeconomic indices are consistent with a causal role of birth weight.

Finally, in line with observational studies showing lifelong negative cognitive consequences for children born with very low birth weight, ${ }^{11}$ this study found evidence supporting the hypothesis that the contribution of birth weight to intelligence may be causal. Additionally, as previous studies mainly focused on children with very low birth weight, our findings add to the literature by replicating these results in a sample of children with birth weight mostly within the normal range. Taken together, available evidence on the association between birth weight and cognitive outcomes suggests that compensation effects of cognitive abilities for children born with low birth weight would not be able to fully counteract the negative effects of low birth weight. ${ }^{54}$

\section{Implications}

Future studies should attempt to clarify the putative causal mechanisms explaining the associations that we found. It has been suggested that restricted fetal growth has a negative impact on brain development ${ }^{55}$ and that this might be a mechanism explaining part of the association between birth weight and mental health and socioeconomic outcomes. For example, a study found alterations in the brain's reactive system and white matter in very low birth-weight children, which was associated with lowered fluid intelligence and heightened anxiety. ${ }^{55}$ Future studies using quasi-experimental designs should be conducted to establish whether brain development lays on the causal path between birth weight and psychosocial outcomes, as well as to identify the brain regions implicated, which may differ across outcomes. Similarly, environmental mechanisms should be identified, as they might be potential targets for interventions aiming to promote mental and socioeconomic well-being among low birth-weight children.

\section{Limitations}

First, the phenotypes considered in this study rely on the definitions and samples used in the original GWASs, which are often highly heterogeneous regarding the recruited population, the definition of the phenotype and the assessment. Although this heterogeneity results from the need to use very large samples to identify small genetic effects, it may also influence our findings. However, studies such as those conducted in independent samples using polygenic scores derived from these GWASs seem to corroborate the validly of their phenotypes. Second, owing to data availability, this study is limited to individuals of European ancestry. Third, because a large proportion of individuals included in the birthweight GWASs had a birth weight within the normal range, the results of our analyses might not reflect the effect of extremely low/high birth weight on mental health, cognitive and social outcomes. Additionally, our analyses assume a linear relation between birth weight and outcomes. ${ }^{26,49}$ Fourth, we could not explore potential gender differences in the association between birth weight and mental health, as gender-specific GWAS summary statistics were not available. Fifth, although we conducted a large array of sensitivity analyses showing the robustness of our findings, horizontal pleiotropy cannot be completely ruled out, as the biological action of most included SNPs is not fully understood yet. Sixth, most of the reported associations only concerned adults and they may differ during other developmental periods. Seventh, although our analyses took into account the correlated role of maternal genotype, residual confounding dynastic effects cannot be excluded, including those related to paternal effects. ${ }^{23}$ Future studies including both maternal and paternal genotype, as well as studies based on within-family GWASs (currently not largely available but necessary to go beyond the assumptions of between-family Mendelian randomisation designs) ${ }^{56}$ are needed to corroborate our results. $^{57}$

Massimiliano orri (D, PhD, McGill Group for Suicide Studies, Douglas Mental Health Research Institute, Department of Psychiatry, McGill University, Montreal, Canada; and Bordeaux Population Health Research Centre, Inserm U1219, University of Bordeaux, France; Jean-Baptiste Pingault, PhD, Division of Psychology and Language Sciences, Department of Clinical, Educational and Health Psychology, University College London; and Social Genetic and Developmental Psychiatry Centre, King's College London, UK; Research Institute, Department of Psychiatry, McGill University, Montreal, Canada; Anne-Monique Nuyt, MD, Centre Hospitalier Universitaire Sainte-Justine Research Center, Department of Pediatrics, University of Montreal, Canada; Richard E. Tremblay, PhD, Department of Pediatrics and Psychology, University of Montreal, Canada; and School of Public Health, University College Dublin, Ireland; Sylvana M. Côté, PhD, Bordeaux Population Health Research Centre, Inserm U1219, University of Bordeaux, France; and Department of Social and Preventive Medicine, School of Public Health, University of Montreal, Canada; Marie-Claude Geoffroy (D), PhD, McGill Group for Suicide Studies, Douglas Mental Health Research Institute, Department of Psychiatry, McGill University, Montreal; and Department of Education and Counselling Psychology, McGill University, Montreal, Canada

Correspondence: Massimiliano Orri. Email: massimiliano.orri@mail.mcgill.ca

First received 13 Mar 2020, final revision 15 Dec 2020, accepted 16 Dec 2020

\section{Supplementary material}

Supplementary material is available online at https://doi.org/10.1192/bjp.2021.15.

$$
\text { Data availability }
$$

This study is based on publicly available summary statistics. 


\section{Author contributions}

MO designed the study, performed data analyses, interpreted the data and drafted the manuscript. J.-B.P. contributed to the study design, data analysis, data interpretation and writing of the final manuscript. All authors contributed to data interpretation and writing of the manuscript.

\section{Funding}

This project has received funding from the European Union's Horizon 2020 research and innovation programme under the Marie Skłodowska-Curie grant agreement no. 793396

\section{Declaration of interest}

J.-B.P. is a fellow of MQ: Transforming Mental Health (MQ16IP16). G.T. holds a Canada Research Chair (Tier 1) and a NARSAD Distinguished Investigator Award and is supported by grants from the Canadian Institute of Health Research (CIHR) (FDN148374 and EGM141899). M.-C. G. and G.T. are supported by the Fonds de recherche du Québec - Santé (FRQS) through the Quebec Network on Suicide, Mood Disorders and Related Disorders. M.-C.G. holds a Canada Research Chair (Tier 2), receives funding from the American Foundation for Suicide Prevention and is a fellow of the Fonds de Recherche du Québec. Funders have no role in the study design, data analysis, interpretation of the data or writing of the manuscript.

\section{References}

1 Abel KM, Wicks S, Susser ES, Dalman C, Pedersen MG, Mortensen PB, et al. Birth weight, schizophrenia and adult mental disorder: is risk confined to the smallest babies? Arch Gen Psychiatry 2010; 67: 923-30.

2 Franz AP, Bolat GU, Bolat H, Matijasevich A, Santos IS, Silveira RC, et al. Attention-deficit/hyperactivity disorder and very preterm/very low birth weight: a meta-analysis. Pediatrics 2018; 141: e20171645

3 Gunnell D, Rasmussen F, Fouskakis D, Tynelius P, Harrison G. Patterns of fetal and childhood growth and the development of psychosis in young males: a cohort study. Am J Epidemiol 2003; 158: 291-300.

4 Lampi KM, Lehtonen L, Tran PL, Suominen A, Lehti V, Banerjee PN, et al. Risk of autism spectrum disorders in low birth weight and small for gestational age infants. J Pediatr 2012; 161: 830-6.

5 Loret de Mola C, de França GVA, de Quevedo LA, Horta BL. Low birth weight, preterm birth and small for gestational age association with adult depression: systematic review and meta-analysis. Br J Psychiatry 2014; 205: 340-7.

6 Nosarti C, Reichenberg A, Murray RM, Cnattingius S, Lambe MP, Yin L, et al. Preterm birth and psychiatric disorders in young adult life. Arch Gen Psychiatry 2012; 69: 610-7.

7 Pettersson E, Larsson H, D'Onofrio B, Almqvist C, Lichtenstein P. Association of fetal growth with general and specific mental health conditions. JAMA Psychiatry 2019; 76: 536-43.

8 Orri M, Gunnell D, Richard-Devantoy S, Bolanis D, Boruff J, Turecki G, et al. Inutero and perinatal influences on suicide risk: a systematic review and metaanalysis. Lancet Psychiatry 2019; 6: 477-92.

9 Strauss RS. Adult functional outcome of those born small for gestational age: twenty-six-year follow-up of the 1970 British Birth Cohort. JAMA 2000; 283 : 625-32.

10 Jelenkovic A, Mikkonen J, Martikainen P, Latvala A, Yokoyama Y, Sund R, et al. Association between birth weight and educational attainment: an individualbased pooled analysis of nine twin cohorts. J Epidemiol Community Health 2018; $72:$ 832-7.

11 Flensborg-Madsen T, Mortensen EL. Birth weight and intelligence in young adulthood and midlife. Pediatrics 2017; 139: e20163161.

12 O'Donnell KJ, Meaney MJ. Fetal origins of mental health: the developmental origins of health and disease hypothesis. Am J Psychiatry 2017; 174: 319-28.

13 Schlotz W, Phillips DIW. Fetal origins of mental health: evidence and mechanisms. Brain Behav Immun 2009; 23: 905-16.

14 Thompson BL, Levitt $P$, Stanwood GD. Prenatal exposure to drugs: effects on brain development and implications for policy and education. Nat Rev Neurosci 2009; 10: 303-12

15 Lewis AJ, Austin E, Galbally M. Prenatal maternal mental health and fetal growth restriction: a systematic review. J Dev Orig Health Dis 2016; 7: 416-28.

16 Aizer A, Currie J. The intergenerational transmission of inequality: maternal disadvantage and health at birth. Science 2014; 344: 856-61.

17 Dessì A, Corona L, Pintus R, Fanos V. Exposure to tobacco smoke and low birth weight: from epidemiology to metabolomics. Expert Rev Proteomics 2018; 15 $647-56$.

18 Lanphear BP, Vorhees CV, Bellinger DC. Protecting children from environmental toxins. PLOS Med 2005; 2: e61.
19 Englund-Ögge L, Brantsæter AL, Juodakis J, Haugen M, Meltzer HM, Jacobsson B, et al. Associations between maternal dietary patterns and infant birth weight, small and large for gestational age in the Norwegian Mother and Child Cohort Study. Eur J Clin Nutr 2019; 73: 1270-82.

20 Orri M, Russell A, Mars B, Turecki G, Gunnell D, Heron J, et al. Perinatal adversity profiles and suicide attempt in adolescence and young adulthood: Iongitudinal analyses from two 20-year birth cohorts. Psychol Med [Epub ahead of print] 6 Oct 2020. Available from: https://doi.org/10.1017/S0033291720002974.

21 Burgess S, Butterworth A, Thompson SG. Mendelian randomization analysis with multiple genetic variants using summarized data. Genet Epidemiol 2013; 37: 658-65.

22 Davies NM, Holmes MV, Davey Smith G. Reading Mendelian randomisation studies: a guide, glossary and checklist for clinicians. BMJ 2018; 362: k601.

23 Pingault J-B, O'Reilly PF, Schoeler T, Ploubidis GB, Rijsdijk F, Dudbridge F. Using genetic data to strengthen causal inference in observational research. Nat Rev Genet 2018; 19: 566-80.

24 Arafat S, Minică CC. Fetal origins of mental disorders? An answer based on Mendelian randomization. Twin Res Hum Genet 2018; 21: 485-94.

25 Warrington NM, Freathy RM, Neale MC, Evans DM. Using structural equation modelling to jointly estimate maternal and fetal effects on birthweight in the UK Biobank. Int J Epidemiol 2018; 47: 1229-41.

26 Warrington NM, Beaumont RN, Horikoshi M, Day FR, Helgeland Ø, Laurin C, et al. Maternal and fetal genetic effects on birth weight and their relevance to cardio-metabolic risk factors. Nat Genet 2019; 51: 804

27 Gage SH, Munafò MR, Davey Smith G. Causal inference in developmental origins of health and disease (DOHaD) Research. Annu Rev Psychol 2016; 67: 567-85.

28 Burgess S, Thompson SG. Avoiding bias from weak instruments in Mendelian randomization studies. Int J Epidemiol 2011; 40: 755-64.

29 Demontis D, Walters RK, Martin J, Mattheisen M, Als TD, Agerbo E, et al Discovery of the first genome-wide significant risk loci for attention deficit/ hyperactivity disorder. Nat Genet 2019; 51: 63-75.

30 Grove J, Ripke S, Als TD, Mattheisen M, Walters RK, Won H, et al. Identification of common genetic risk variants for autism spectrum disorder. Nat Genet 2019; 51: 431-44.

31 Stahl EA, Breen G, Forstner AJ, McQuillin A, Ripke S, Trubetskoy V, et al. Genome-wide association study identifies 30 loci associated with bipolar disorder. Nat Genet 2019: 51: 793-803.

32 Wray NR, Ripke S, Mattheisen M, Trzaskowski M, Byrne EM, Abdellaoui A, et al. Genome-wide association analyses identify 44 risk variants and refine the genetic architecture of major depression. Nat Genet 2018; 50: 668-81.

33 International Obsessive Compulsive Disorder Foundation Genetics Collaborative (IOCDF-GC), OCD Collaborative Genetics Association Studies (OCGAS). Revealing the complex genetic architecture of obsessive-compulsive disorder using meta-analysis. Mol Psychiatry 2018; 23: 1181-8.

34 Duncan LE, Ratanatharathorn A, Aiello AE, Almli LM, Amstadter AB, Ashley-Koch AE, et al. Largest GWAS of PTSD $(N=20070)$ yields genetic overlap with schizophrenia and sex differences in heritability. Mol Psychiatry 2018; 23: 666-73.

35 Pardiñas AF, Holmans P, Pocklington AJ, Escott-Price V, Ripke S, Carrera N et al. Common schizophrenia alleles are enriched in mutation-intolerant genes and in regions under strong background selection. Nat Genet 2018; 50: 381-9.

36 Erlangsen A, Appadurai V, Wang Y, Turecki G, Mors O, Werge T, et al. Genetics of suicide attempts in individuals with and without mental disorders: a populationbased genome-wide association study. Mol Psychiatry 2020; 25: 2410-21.

37 Savage JE, Jansen PR, Stringer S, Watanabe K, Bryois J, de Leeuw CA, et al. Genome-wide association meta-analysis in 269,867 individuals identifies new genetic and functional links to intelligence. Nat Genet 2018; 50: 912-9.

38 Lee JJ, Wedow R, Okbay A, Kong E, Maghzian O, Zacher M, et al. Gene discovery and polygenic prediction from a genome-wide association study of educational attainment in 1.1 million individuals. Nat Genet 2018; 50: 1112-21.

39 Hill WD, Hagenaars SP, Marioni RE, Harris SE, Liewald DCM, Davies G, et al. Molecular genetic contributions to social deprivation and household income in UK Biobank. Curr Biol 2016; 26: 3083-9.

40 R Core Team. R: A Language and Environment for Statistical Computing. R Foundation for Statistical Computing, 2016 (https://www.R-project.org/).

41 Hemani G, Zheng J, Elsworth B, Wade KH, Haberland V, Baird D, et al. The MRBase platform supports systematic causal inference across the human phenome. elife 2018; 7: e34408.

42 Yavorska OO, Burgess S. MendelianRandomization: an R package for performing Mendelian randomization analyses using summarized data. Int $\mathrm{J}$ Epidemiol 2017; 46: 1734-9.

43 Bowden J, Davey Smith G, Burgess S. Mendelian randomization with invalid instruments: effect estimation and bias detection through Egger regression. Int J Epidemiol 2015; 44: 512-25 
44 Zhao Q, Wang J, Hemani G, Bowden J, Small DS. Statistical inference in twosample summary-data Mendelian randomization using robust adjusted profile score. Ann Statist 2020; 48: 1742-69.

45 Verbanck M, Chen C-Y, Neale B, Do R. Detection of widespread horizontal pleiotropy in causal relationships inferred from Mendelian randomization between complex traits and diseases. Nat Genet 2018; 50: 693-8.

46 Breslau N. Psychiatric sequelae of low birth weight. Epidemiol Rev 1995; 17 96-106.

47 Galéra C, Côté SM, Bouvard MP, Pingault J-B, Melchior M, Michel G, et al. Early risk factors for hyperactivity-impulsivity and inattention trajectories from age 17 months to 8 years. Arch Gen Psychiatry 2011; 68: 1267-75.

48 Lim KX, Liu C-Y, Schoeler T, Cecil CAM, Barker ED, Viding E, et al. The role of birth weight on the causal pathway to child and adolescent ADHD symptomatology: a population-based twin differences longitudinal design. J Child Psychol Psychiatry 2018; 59: 1036-43.

49 Class QA, Rickert ME, Larsson H, Lichtenstein P, D'Onofrio BM. Fetal growth and psychiatric and socioeconomic problems: population-based sibling comparison. Br J Psychiatry 2014; 205: 355-61.

50 Turecki G, Brent DA. Suicide and suicidal behaviour. Lancet 2016; 387: 1227-39.
51 Gunnell D. A population health perspective on suicide research and prevention. Crisis 2015; 36: 155-60.

52 Kajantie E. Fetal origins of stress-related adult disease. Ann N Y Acad Sci 2006 ; 1083: 11-27.

53 Wojcik W, Lee W, Colman I, Hardy R, Hotopf M. Foetal origins of depression? A systematic review and meta-analysis of low birth weight and later depression. Psychol Med 2013; 43: 1-12.

54 Bergman A, Siegal ML. Evolutionary capacitance as a general feature of complex gene networks. Nature 2003; 424: 549-52.

55 Walhovd KB, Fjell AM, Brown TT, Kuperman JM, Chung Y, Hagler DJ, et al. Longterm influence of normal variation in neonatal characteristics on human brain development. Proc Natl Acad Sci 2012; 109: 20089-94.

56 Davey Smith G, Holmes MV, Davies NM, Ebrahim S. Mendel's laws, Mendelian randomization and causal inference in observational data: substantive and nomenclatural issues. Eur J Epidemiol 2020; 35: 99-111.

57 Davies NM, Howe LJ, Brumpton B, Havdahl A, Evans DM, Davey Smith G. Within family Mendelian randomization studies. Hum Mol Genet 2019; 28: R170-9. 